\title{
BMJ Open National database study of trends in bacteraemia aetiology among children and adults in Japan: a longitudinal observational study
}

\author{
Yoshiki Kusama (D) , ${ }^{1,2}$ Kenta Ito, ${ }^{3}$ Haruhisa Fukuda, ${ }^{4}$ Nobuaki Matsunaga, ${ }^{1}$ \\ Norio Ohmagari ${ }^{1,2}$
}

To cite: Kusama Y, Ito K, Fukuda $\mathrm{H}$, et al. National database study of trends in bacteraemia aetiology among children and adults in Japan: a longitudinal observational study. BMJ Open 2021;11:e043774. doi:10.1136/ bmjopen-2020-043774

- Prepublication history and additional material for this paper are available online. To view these files, please visit the journal online (http://dx.doi. org/10.1136/bmjopen-2020043774).

Received 18 August 2020 Revised 18 March 2021 Accepted 21 March 2021
Check for updates

(C) Author(s) (or their employer(s)) 2021. Re-use permitted under CC BY-NC. No commercial re-use. See rights and permissions. Published by BMJ.

For numbered affiliations see end of article.

Correspondence to

Dr Yoshiki Kusama;

stone.bagle@gmail.com

\section{ABSTRACT}

Objectives Domestic epidemiological studies are needed to ascertain the disease burden of bacteraemia in individual countries. This study aimed to evaluate the domestic trends in paediatric and adult bacteraemia in Japan.

Setting Laboratory-based surveillance was used to obtain data from 592 hospitals located throughout Japan.

Participants The study was conducted using the results of 827780 and 3512524 blood culture tests obtained from children and adults, respectively, between January 2010 and December 2016.

Outcome measures We analysed the temporal trends in specific bacterial species (Staphylococcus aureus, Streptococcus pneumoniae, Streptococcus pyogenes, Streptococcus agalactiae, Escherichia coli, Klebsiella pneumoniae, Salmonella enterica, Haemophilus influenzae, Listeria monocytogenes and Neisseria meningitidis) detected from the blood cultures of children and adults. For children aged $\leq 2$ years, the data were analysed for each year of age. The proportions of bacteraemia-positive results among the patients were also evaluated.

Results The number of bacteraemia-positive samples over the study period was $47,125 / 827,855(5.7 \%)$ in children and 959,765/3,513,885 (27.3\%) in adults. $S$. pneumoniae was the most frequent cause of bacteraemia in children in 2010. However, after 2011, S. aureus bacteraemia was the most frequent, followed by $S$. pneumoniae and E. coli. E. coli bacteraemia showed significant increases in both children and adults. In children, $S$. pneumoniae and $H$. influenzae bacteraemia decreased from 2010 to 2013. However, S. pneumoniae bacteraemia case numbers stabilised from 2013 , whereas $H$. influenzae bacteraemia cases continued to decrease until 2016.

Conclusion The results suggest that the introduction of the 13-valent pneumococcal conjugate vaccine did not substantially affect disease occurrence. In contrast to the decreasing trends in $H$. influenzae and $S$. pneumoniae bacteraemia, $S$. aureus, E. coli and $S$. agalactiae bacteraemia showed increasing trends. These findings shed light on recent temporal trends in bacteraemia in both children and adults in Japan.

\section{Strengths and limitations of this study}

- This is the first study to document the aetiological changes in bacteraemia in Japan following the widespread dissemination of immunisation.

- The study data were obtained from Japan's largest government-operated microbiological surveillance system, which increases the findings' national generalisability.

- The separate evaluation of bacteraemia in children and adults due to their aetiological differences simplifies the interpretation of the results.

- The data lacked important patient-level information, such as inpatient or outpatient statuses, patient background and prognoses.

- Causative pathogen subtypes, such as capsule types and serotypes, could not be analysed due to database limitations.

\section{INTRODUCTION}

Bacteraemia continues to impose a considerable disease burden in both lower-income and higher-income countries. ${ }^{12}$ Due to international differences in the distribution of causative bacteria (eg, meningococci and non-typhoidal salmonella), ${ }^{3}{ }^{4}$ individual countries need to conduct domestic epidemiological studies to ascertain disease burden and inform the development of targeted strategies to improve public health. Studies from various countries have examined the changes in paediatric bacteraemia following the spread of immunisation and improvements in sanitation. ${ }^{5-7}$ For example, the implementation of a universal pneumococcal immunisation programme in a US integrated managed care consortium substantially reduced the morbidity of bacteraemia in young children, with contaminants more frequently recovered from samples than true pathogens. ${ }^{5}$ Furthermore, these vaccinations shifted the burden of paediatric bacteraemia 
in the USA from pneumococcal infections to those by Staphylococcus aureus, Salmonella spp, and Escherichia coli. ${ }^{5}$ Other studies have also documented the epidemiological changes in bacteraemia in adult populations. ${ }^{89}$

In Japan, the Haemophilus influenzae type b vaccine and 7-valent pneumococcal conjugate vaccine were introduced as 'optional' vaccines in December 2008 and February 2010, respectively. The early dissemination of these vaccines may have been stifled as optional vaccines require patient out-of-pocket payments under Japan's healthcare system. However, the Ministry of Health, Labour and Welfare (MHLW) implemented an extensive immunisation promotion programme in November 2010 and approved these two vaccines as 'mandatory' (removing the need for out-of-pocket payments) in April 2013, thereby increasing vaccine utilisation. In November 2013, the 7-valent pneumococcal conjugate vaccine was replaced by the 13-valent version. Despite these changes in vaccine availability and coverage, their effects on the epidemiology of paediatric bacteraemia in Japan remain unclear. Ubukata et al analysed patients with invasive pneumococcal diseases between 2010 and 2017 using a laboratory-based surveillance system, and reported that the causative pathogens demonstrated serotype changes over time. ${ }^{10}$ However, their surveillance system was not government-operated and participation was voluntary, and their findings may be susceptible to selection bias. In addition, little is known about the epidemiological changes in non-pneumococcal bacteraemia in Japan.

Through an analysis of data from the largest governmentoperated microbiological surveillance system in Japan, this study aimed to evaluate domestic trends in paediatric and adult bacteraemia, including the epidemiological changes in H. influenzae and pneumococcal bacteraemia.

\section{METHODS}

\section{Data source}

We conducted the observational database study. We acquired information on the results of blood culture tests conducted between January 2010 and December 2016 from a database provided by the MHLW's Japan Nosocomial Infections Surveillance (JANIS) Clinical Laboratory Division, which routinely collects microbiological test results from approximately 2000 hospitals located throughout the country. In 2019, 24.8\% of all hospitals $(2,075 / 8372)$. Participation was high among those with $>500$ beds $(82.8 \%, 338 / 408)$, while it was relatively low among hospitals containing between 200 and 499 $(46.9 \%, 1019 / 2174)$ and <200 beds $(12.4 \%, 718 / 5790){ }^{11}$ To eliminate the effects caused by temporal changes in the number of participating hospitals on the number of blood culture samples, we only included data from facilities that continuously participated in JANIS throughout the study period. Because JANIS aims to provide microbiological information from a diverse range of hospitals (including smaller, resource-limited hospitals), the programme only collects basic information to encourage sustainable participation. Thus, the database did not include detailed information on causative pathogen subtypes (eg, H. influenzae capsule types or Streptococcus pneumoniae serotypes).

\section{Data processing}

We compiled the blood culture results as 'sets' for each patient, which included the combination of aerobic and anaerobic cultures if both samples were obtained. To eliminate data duplication from simultaneously obtained blood cultures (ie, multiple sets) and blood cultures obtained to confirm microbiological cure (ie, follow-up cultures), we only examined the first positive result in patients who had $\geq 2$ blood cultures in a year. If all the blood cultures obtained from a patient in a year were negative, we only included the first negative result.

\section{Outcomes}

We first evaluated the proportions of bacteraemia-positive results from blood cultures in children and adults. For this study, age was provided in 5-year increments. As a consequence, children were defined as individuals aged $<20$ years, which differs from the definition ( $<18$ years) used by the United Nations. ${ }^{12}$ Adults were defined as individuals aged $\geq 20$ years.

Next, we analysed the temporal trends in bacterial species detected from the blood cultures of children and adults. This analysis included the following bacteria, which are known to cause bloodstream infections in healthy individuals: S. aureus, S. pneumoniae, Streptococcus pyogenes, Streptococcus agalactiae, E. coli, Klebsiella pneumoniae, Salmonella enterica, $H$. influenzae, Listeria monocytogenes and Neisseria meningitidis. We excluded bacteria that generally cause bloodstream infections in immunocompromised patients or are regarded as culture contaminants, such as Pseudomonas aeruginosa, Bacillus spp, and coagulase-negative staphylococci. For children aged $\leq 2$ years, the data were analysed for each year of age due to the higher risk of bloodstream infections. ${ }^{5}$

\section{Statistical analysis}

To evaluate the bacteria-specific temporal changes in bacteraemia over the study period, we examined the trends in the proportions of bacteria-specific bacteraemia to the total number of tested blood cultures using the Cochran-Armitage trend test. All data management and statistical analyses were conducted using R V.4.0.2 (R Foundation for Statistical Computing, Vienna, Austria). Two-tailed $\mathrm{p}$ values $<0.05$ were considered statistically significant.

\section{Patient and public involvement}

No patients or members of the public were involved in the development or conduct of the study.

The board waived the need for informed consent as the data were anonymised before being received by the authors. 
Table 1 Annual numbers of bacteraemia cases according to causative bacteria in children and adults

\begin{tabular}{|c|c|c|c|c|c|c|c|c|}
\hline \multirow[b]{2}{*}{ Paediatric population } & \multicolumn{7}{|c|}{ No of bacteraemia cases/year } & \multirow{2}{*}{$\begin{array}{l}\text { Cochran-Armitage } \\
\text { test ( } p \text { value) }\end{array}$} \\
\hline & 2010 & 2011 & 2012 & 2013 & 2014 & 2015 & 2016 & \\
\hline $\begin{array}{l}\text { Streptococcus } \\
\text { pneumoniae }\end{array}$ & 891 & 574 & 382 & 337 & 325 & 360 & 330 & $<0.0001$ \\
\hline Staphylococcus aureus & 648 & 628 & 668 & 740 & 708 & 743 & 724 & $<0.0001$ \\
\hline Escherichia coli & 259 & 287 & 284 & 300 & 299 & 326 & 375 & $<0.0001$ \\
\hline Klebsiella pneumoniae & 112 & 99 & 116 & 105 & 105 & 112 & 120 & 0.0152 \\
\hline Salmonella enterica & 27 & 23 & 33 & 33 & 59 & 70 & 56 & $<0.0001$ \\
\hline Haemophilus influenzae & 412 & 195 & 84 & 53 & 36 & 43 & 28 & $<0.0001$ \\
\hline Streptococcus pyogenes & 20 & 36 & 20 & 34 & 22 & 54 & 39 & $<0.0001$ \\
\hline $\begin{array}{l}\text { Streptococcus } \\
\text { agalactiae }\end{array}$ & 112 & 119 & 148 & 129 & 158 & 160 & 172 & $<0.0001$ \\
\hline Listeria monocytogenes & 3 & 5 & 6 & 4 & 8 & 7 & 9 & 0.0281 \\
\hline Neisseria meningitidis & 0 & 4 & 0 & 3 & 0 & 2 & 2 & 0.6182 \\
\hline Others & 5.904 & 4984 & 4631 & 4279 & 4265 & 4948 & 4725 & - \\
\hline Total & 8388 & 6954 & 6372 & 6017 & 5989 & 6825 & 6580 & - \\
\hline Adult population & 2010 & 2011 & 2012 & 2013 & 2014 & 2015 & 2016 & $\begin{array}{l}\text { Cochran-Armitage } \\
\text { test ( } P \text { value) }\end{array}$ \\
\hline S. pneumoniae & 1470 & 1553 & 1551 & 1584 & 1682 & 1654 & 1626 & $<0.0001$ \\
\hline S. aureus & 13239 & 13334 & 13390 & 13011 & 13377 & 13783 & 14444 & $<0.0001$ \\
\hline E. coli & 16906 & 18764 & 21238 & 24113 & 26819 & 29728 & 32935 & $<0.0001$ \\
\hline K. pneumoniae & 6517 & 7496 & 8305 & 9229 & 10103 & 10746 & 11646 & $<0.0001$ \\
\hline S. enterica & 179 & 130 & 157 & 151 & 181 & 168 & 196 & 0.0601 \\
\hline H. influenzae & 166 & 160 & 182 & 202 & 222 & 233 & 283 & 0.0131 \\
\hline S. pyogenes & 315 & 454 & 484 & 462 & 503 & 559 & 650 & 0.0005 \\
\hline S. agalactiae & 1085 & 1206 & 1306 & 1461 & 1534 & 1665 & 1893 & $<0.0001$ \\
\hline L. monocytogenes & 88 & 105 & 139 & 181 & 185 & 205 & 211 & $<0.0001$ \\
\hline N. meningitidis & 7 & 2 & 4 & 7 & 14 & 13 & 13 & 0.0219 \\
\hline Others & 77596 & 75992 & 78732 & 84333 & 89131 & 94793 & 101584 & \\
\hline Total & 117568 & 119196 & 125488 & 134734 & 143751 & 153547 & 165481 & \\
\hline
\end{tabular}

\section{RESULTS}

Data were obtained from 592 hospitals. In children, we obtained blood culture results from 98295 samples in 2010, 109611 samples in 2011, 115172 samples in 2012, 115172 samples in 2013, 120561 samples in 2014, 131297 samples in 2015 and 138452 samples in 2016. In adults, we obtained blood culture results from 390796 samples in 2010, 428529 samples in 2011, 462153 samples in 2012, 497143 samples in 2013, 530399 samples in 2014, 579195 samples in 2015, and 625670 samples in 2016. Overall, the number of bacteraemia-positive samples was 47125 $(5.7 \%)$ in children and $959765(27.3 \%)$ in adults.

Table 1 shows the annual numbers of bacteraemia cases according to causative bacteria, and figure 1 shows the temporal trends in the number of bacteraemia cases for six major pathogens (S. pneumoniae, S. aureus, E. coli, K. pneumoniae, H. influenzae and S. agalactiae). S. pneumoniae was the most frequent cause of bacteraemia in children in 2010. However, after 2011, S. aureus bacteraemia was the most frequent in children, followed by S. pneumoniae and E. coli. In 2016, E. coli bacteraemia became more common than S. pneumoniae bacteraemia. In contrast, E. coli bacteraemia was the most frequent in adults, followed by $S$. aureus and K. pneumoniae, consistently. E. coli bacteraemia significantly increased in both children and adults. An increase in $S$. enterica bacteraemia was observed in children, but not adults, during the study period. S. agalactiae bacteraemia increased in both children and adults. L. monocytogenes was rarely detected in children, but accounted for more than 100 bacteraemia cases in adults in all years except 2010; in addition, L. monocytogenes bacteraemia increased throughout the study period in adults. In contrast, $N$. meningitidis was rarely detected in both children and adults. In children, both $S$. pneumoniae and $H$. influenzae bacteraemia decreased from 2010 to 2013. However, S. pneumoniae bacteraemia cases were relatively consistent from 2013, whereas $H$. influenzae bacteraemia cases continued to decrease and were almost eliminated by 2016 . 

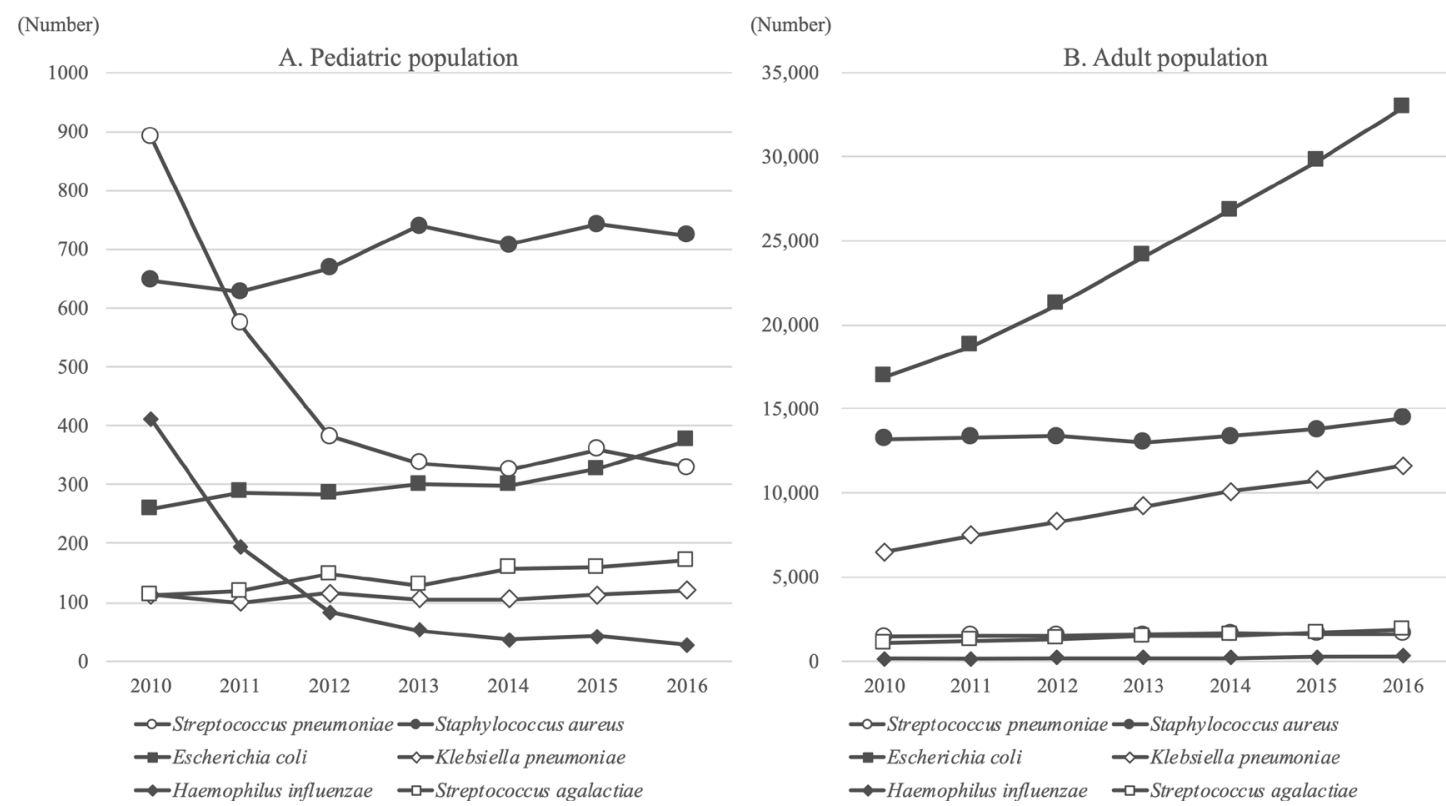

Figure 1 Temporal trends in the number of bacteraemia cases according to causative bacteria. (A) paediatric population. (B) adult population.

The breakdown of bacteria-specific bacteraemia trends in children aged 0,1 and 2 years is shown in figure 2. Although the number of $S$. aureus bacteraemia cases was highest in 0 -year-old children, numerous cases were also observed in 1-year-old and 2-year-old children. A decrease in the number of S. pneumoniae bacteraemia cases from 2010 to 2013 was observed in all three age groups; however, these cases became relatively stable in 0-year-old and 1-year-old children, but increased in 2-year-old children.

\section{DISCUSSION}

To the best of our knowledge, this is the largest study that evaluates the trends and aetiology of bacteraemia in children and adults in Japan. Because the data were obtained from the country's largest government-operated microbiological surveillance system, our findings may be regarded as having a high degree of generalisability to the Japanese population.

Our analysis revealed reductions in $S$. pneumoniae and $H$. influenzae bacteraemia over the study period, but increases in $S$. aureus, E. coli, and $S$. agalactiae bacteraemia. In particular, E. coli bacteraemia increased in both children and adults, with the number of adult cases doubling over the 6-year study period. Similar increases in E. coli bacteraemia have also been reported in the UK, Europe and the USA. ${ }^{813}$ Although the reasons remain unclear, E. coli bacteraemia frequently originates from urinary tract infections. ${ }^{1415}$ Other reports have found
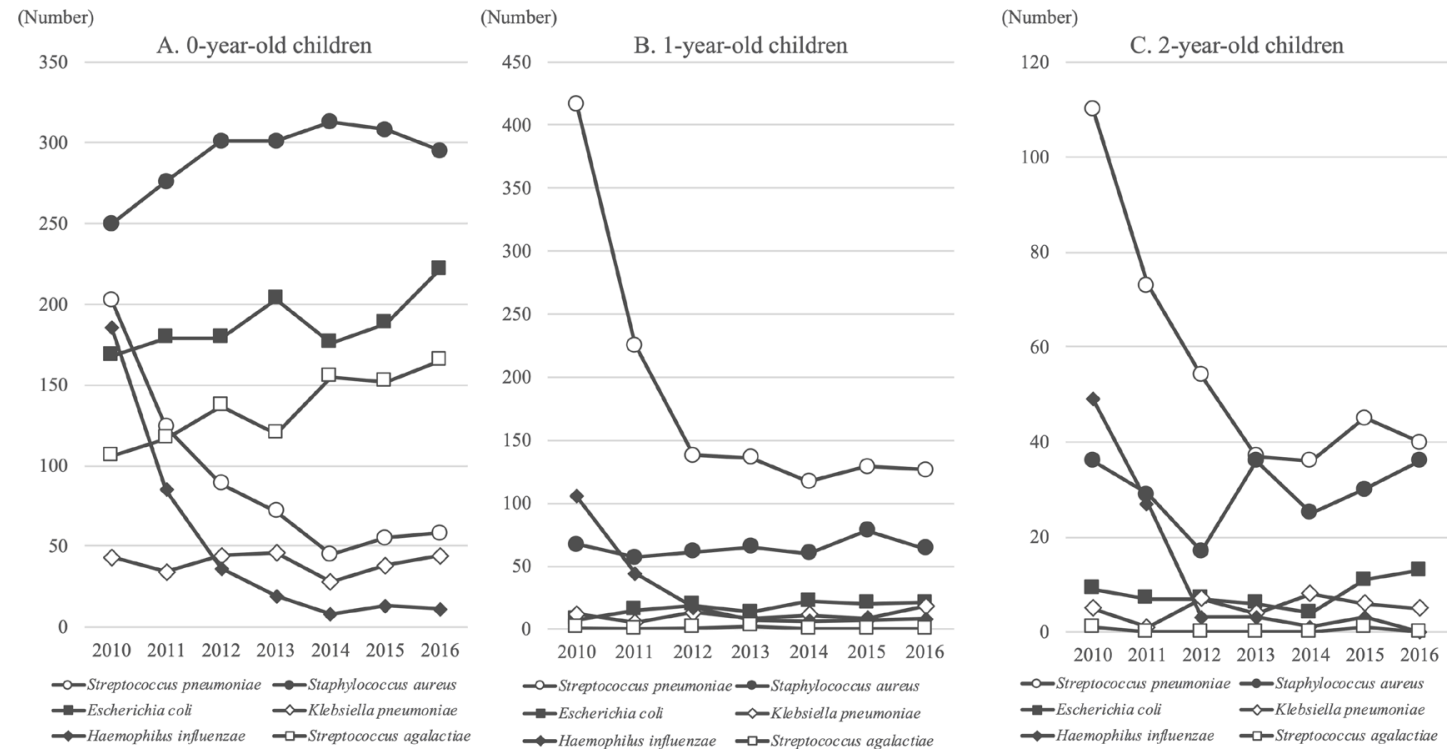

Figure 2 Temporal trends in the number of bacteraemia cases according to causative bacteria in children aged 0-2 years (A) 0 -year-old children. (B) 1-year-old children. (C) 2-year-old children. 
that community-onset cases account for a greater proportion of $E$. coli bacteraemia than hospital-onset cases and antimicrobial-resistant strains are increasing. ${ }^{8}{ }^{15}$ While population ageing is a possible factor for these increases in adults, ${ }^{16}$ it does not explain the rise in paediatric cases. Although there is currently no definitive evidence, it has been postulated that the emergence of antimicrobial resistance is a bacterial factor that contributed to the increase in $E$. coli bacteraemia within the community. ${ }^{8}{ }^{85}$ Similarly, a study reported that the rise in a pandemic $E$. colistrain was associated with an increase in infant urinary tract infections. ${ }^{17}$ Further investigations are needed to elucidate these issues.

S. aureus was a major cause of bacteraemia among both adults and children in our study population. Studies from other countries have also reported that $S$. aureus bacteraemia is likely to occur in the hospital setting and in infants. ${ }^{18} 19$ Therefore, the high incidence of $S$. aureus bacteraemia in our subjects may be explained by the focus on hospitalised patients (including those in neonatal intensive care units). Similar results have also been reported in a metropolitan hospital in Australia. ${ }^{20}$ There may be a need for programmes to promote hospital infection control and prevention measures, such as compliance with hand hygiene guidelines, to halt the increase in paediatric $S$. aureus bacteraemia. Although studies have also reported increases in community-onset $S$. aureus bacteraemia, ${ }^{18} 21$ it was unclear as to whether our cases originated in the community or healthcare setting as our data did not specify the location of onset. Our observed increases in S. agalactiae bacteraemia were corroborated by previous studies that reported increases in late-onset infections. $^{22} 23$

Immunisations using the $H$. influenzae type $\mathrm{b}$ vaccine and pneumococcal conjugate vaccine have increased rapidly in Japan, with almost all eligible children receiving these inoculations since 2013 (online supplemental table 1). H. influenzae bacteraemia cases continued to decline over the study period, but the decline in $S$. pneumoniae bacteraemia cases ceased in 2013. Among 1-year-old children (who are most eligible for these immunisations), both bacteraemia types decreased sharply until 2012, whereupon the decrease in pneumococcal bacteraemia stopped. Numerous studies in other countries have demonstrated the efficacy of the 13-valent pneumococcal conjugate vaccine against pneumococcal bacteraemia, ${ }^{54-26}$ but the impact of this vaccine (introduced in November 2013) was not apparent in Japan. We posit that this may have been affected by the higher rate of serotype replacements in pneumococcal bacteraemia in Japan relative to other high-income countries. ${ }^{10}$ Hence, 13-valent vaccine possibly suppressed the increase in bacteraemia causes by 7-valent vaccine-uncovered pneumococcal serotypes. Additionally, S. pneumoniae serotype $12 \mathrm{~F}$ has caused domestic outbreaks of invasive pneumococcal diseases in Japan since 2016, and our observed increase in pneumococcal bacteraemia cases in 2016 may be associated with these outbreaks. ${ }^{27} 28$
L. monocytogenes bacteraemia was observed to be more common in adults than in children among our subjects. Similar findings were reported in the USA, which raised questions about the necessity of $L$. monocytogenes coverage in empiric antibiotic therapy for infants. ${ }^{6}$ However, we observed a consistent-although low-level of L. monocytogenes bacteraemia in Japanese children every year, and the removal of $L$. monocytogenes coverage would require careful consideration. In contrast, the rarity of N. meningitidis bacteraemia in both children and adults suggests that there is currently no need for a mandatory meningococcal vaccine programme in Japan. ${ }^{29}$

Recent years have seen an increased understanding in physicians of the importance of obtaining and testing blood culture samples in Japan, and this was reflected in the rising number of samples taken from both children and adults throughout the study period (online supplemental figure 1). In children, the proportion of bacteraemia-positive cases had gradually declined over time due to the increase in the number of bacteraemianegative samples; the latest proportion of bacteraemiapositive cases in 2016 was $4.75 \%$. The proportion of bacteraemia-positive cases due to our target pathogens (ie, bacteria that can cause bacteraemia in healthy children) was $1.30 \%$, which is generally higher than those of other high-income countries. ${ }^{5031}$ This disparity may be partially affected by our focus on more severe cases that require hospitalisation. Unfortunately, our database did not include information on the ward in which each positive blood culture was obtained, and we could not identify and exclude patients in intensive care units. Also, increases in the actual number of bacteraemia-positive cases were observed in adults, but not in children. This may have been influenced by the epidemiologic changes in paediatric bacteraemia over the study period.

\section{LIMITATIONS}

Our study has several limitations. First, the JANIS data did not include patient and clinical information such as inpatient or outpatient statuses, patient background and prognoses. As a result, the identification of bacteraemiapositive samples lacked context for interpretation. Second, we attempted to minimise the possible statistical issues stemming from the voluntary nature of the surveillance programme through the exclusion of multiple-set and follow-up blood cultures, but could not address the bias arising from the increasing emphasis on obtaining blood cultures. Third, we assessed the epidemiology of $H$. influenzae and S. pneumoniae bacteraemia, but could not analyse their respective capsule types or serotypes. Further studies are, therefore, needed to evaluate the epidemiological differences, if any, among these various strains. Finally, the relatively high proportion of bacteraemia cases attributed to 'other' bacterial species may be due to blood culture contaminants (eg, skin commensals). However, we were unable to distinguish between contaminants and true pathogens from the laboratory 
data alone. Therefore, the prevalences of E. coli and $S$. aureus bacteraemia cases would be higher if the contaminants could be accurately identified and excluded.

\section{CONCLUSION}

This study described the domestic trends in bacteraemia in Japan from 2010 to 2016. H. influenzae bacteraemia cases continued to decline over the study period, whereas $S$. pneumoniae bacteraemia cases declined until 2013. These findings suggest that the introduction of the 13-valent pneumococcal conjugate vaccine did not have a substantial impact on disease occurrence. In contrast to the decreasing trends in $H$. influenzae and S. pneumoniae bacteraemia, the analysis found increasing trends in $S$. aureus, E. coli and S. agalactiae bacteraemia. Continued epidemiological monitoring is needed to ascertain the changes in the domestic burden of bacteraemia in Japan.

\section{Author affiliations}

${ }^{1}$ AMR Clinical Reference Center, Disease Control and Prevention Center, National Center for Global Health and Medicine, Shinjuku-ku, Tokyo, Japan

${ }^{2}$ Collaborative Chairs Emerging and Reemerging Infectious Diseases, National Center for Global Health and Medicine, Graduate School of Medicine, Tohoku

University, Sendai, Miyagi, Japan

${ }^{3}$ Department of General Pediatrics, Aichi Children's Health and Medical Center, Obu, Aichi, Japan

${ }^{4}$ Department of Health Care Administration and Management, Kyushu University Graduate School of Medical Sciences School of Medicine Department of Health Sciences, Hakata, Fukuoka, Japan

Contributors YK is responsible for the study conceptualisation, design, investigation and drafting of the manuscript. $\mathrm{Kl}$ contributed to the study design, manuscript review and editing and supervision. HF contributed to the study design, data acquisition, formal analysis and supervision. NM contributed to the study design and supervision. NO contributed to the study conceptualisation, funding acquisition and supervision. All authors read and approved the final manuscript.

Funding This work was supported by a research grant from the Ministry of Health, Labour and Welfare of Japan (grant number: 20HA2003).

Competing interests None declared.

Patient consent for publication Not required.

Ethics approval The study was approved by the Kyushu University Institutional Review Board for Clinical Research (approval number: 2019-180).

Provenance and peer review Not commissioned; externally peer reviewed.

Data availability statement Data may be obtained from a third party and are not publicly available. For the JANIS data, the Ministry of Health, Labour and Welfare of Japan has placed strict legal restrictions on the release or sharing of these data. As a result, these data are not publicly available. However, the same data may be obtained from a third party through a formal application.

Supplemental material This content has been supplied by the author(s). It has not been vetted by BMJ Publishing Group Limited (BMJ) and may not have been peer-reviewed. Any opinions or recommendations discussed are solely those of the author(s) and are not endorsed by BMJ. BMJ disclaims all liability and responsibility arising from any reliance placed on the content. Where the content includes any translated material, BMJ does not warrant the accuracy and reliability of the translations (including but not limited to local regulations, clinical guidelines, terminology, drug names and drug dosages), and is not responsible for any error and/or omissions arising from translation and adaptation or otherwise.

Open access This is an open access article distributed in accordance with the Creative Commons Attribution Non Commercial (CC BY-NC 4.0) license, which permits others to distribute, remix, adapt, build upon this work non-commercially, and license their derivative works on different terms, provided the original work is properly cited, appropriate credit is given, any changes made indicated, and the use is non-commercial. See: http://creativecommons.org/licenses/by-nc/4.0/.

\section{ORCID iD}

Yoshiki Kusama http://orcid.org/0000-0002-7072-2338

\section{REFERENCES}

1 Agyeman PKA, Schlapbach LJ, Giannoni E, et al. Epidemiology of blood culture-proven bacterial sepsis in children in Switzerland: a population-based cohort study. Lancet Child Adolesc Health 2017;1:124-33.

2 Fleischmann-Struzek C, Goldfarb DM, Schlattmann P, et al. The global burden of paediatric and neonatal sepsis: a systematic review. Lancet Respir Med 2018;6:223-30.

3 Jafri RZ, Ali A, Messonnier NE, et al. Global epidemiology of invasive meningococcal disease. Popul Health Metr 2013;11:17.

4 GBD 2017 Non-Typhoidal Salmonella Invasive Disease Collaborators. The global burden of non-typhoidal Salmonella invasive disease: a systematic analysis for the global burden of disease study 2017. Lancet Infect Dis 2019;19:1312-24.

5 Greenhow TL, Hung Y-Y, Herz A. Bacteremia in children 3 to 36 months old after introduction of conjugated pneumococcal vaccines. Pediatrics 2017;139:e20162098.

6 Greenhow TL, Hung Y-Y, Herz AM. Changing epidemiology of bacteremia in infants aged 1 week to 3 months. Pediatrics 2012;129:e590-6.

7 Buetti N, Atkinson A, Kottanattu L, et al. Patterns and trends of pediatric bloodstream infections: a 7-year surveillance study. Eur J Clin Microbiol Infect Dis 2017;36:537-44.

8 Vihta K-D, Stoesser N, Llewelyn MJ, et al. Trends over time in Escherichia coli bloodstream infections, urinary tract infections, and antibiotic susceptibilities in Oxfordshire, UK, 1998-2016: a study of electronic health records. Lancet Infect Dis 2018;18:1138-49.

9 de Kraker MEA, Jarlier V, Monen JCM, et al. The changing epidemiology of bacteraemias in Europe: trends from the European antimicrobial resistance surveillance system. Clin Microbiol Infect 2013;19:860-8.

10 Ubukata K, Takata M, Morozumi M, et al. Effects of pneumococcal conjugate vaccine on genotypic penicillin resistance and serotype changes, Japan, 2010-2017. Emerg Infect Dis 2018;24:2010-20.

11 Japan nosocomial infections surveillance. annual open report 2019 (all facilities). Available: https://janis.mhlw.go.jp/english/report/ open_report/2019/3/1/ken_Open_Report_Eng_201900_clsi2012.pdf [Accessed 15 May 2021].

12 United Nations Human Rights. Convention on the rights of the child. Available: https://www.ohchr.org/en/professionalinterest/pages/crc. aspx [Accessed Mar 13, 2020].

13 Spaulding AB, Watson D, Dreyfus J, et al. Epidemiology of bloodstream infections in hospitalized children in the United States, 2009-2016. Clin Infect Dis 2019;69:995-1002.

14 Daga AP, Koga VL, Soncini JGM, et al. Escherichia coli Bloodstream Infections in Patients at a University Hospital: Virulence Factors and Clinical Characteristics. Front Cell Infect Microbiol 2019;9:191.

15 Blandy O, Honeyford K, Gharbi M, et al. Factors that impact on the burden of Escherichia coli bacteraemia: multivariable regression analysis of 2011-2015 data from West London. J Hosp Infect 2019;101:120-8.

16 van der Mee-Marquet NL, Blanc DS, Gbaguidi-Haore H, et al. Marked increase in incidence for bloodstream infections due to Escherichia coli, a side effect of previous antibiotic therapy in the elderly. Front Microbiol 2015;6:646.

17 Cheng M-F, Chen W-L, Hung W-Y, et al. Emergence of extended spectrum- $\beta$-lactamase-producing Escherichia coli O25b-ST131: a major community-acquired uropathogen in infants. Pediatr Infect Dis J 2015;34:469-75.

18 Cobos-Carrascosa E, Soler-Palacín P, Nieves Larrosa M, et al. Staphylococcus aureus bacteremia in children: changes during eighteen years. Pediatr Infect Dis J 2015;34:1329-34.

19 Laupland KB, Lyytikäinen O, Søgaard M, et al. The changing epidemiology of Staphylococcus aureus bloodstream infection: a multinational population-based surveillance study. Clin Microbiol Infect 2013;19:465-71.

20 Shakur SM, Whitehall J, Mudgil P. Pediatric bloodstream infections in metropolitan Australia. World J Pediatr 2019;15:161-7.

21 Iwamoto M, Mu Y, Lynfield R, et al. Trends in invasive methicillinresistant Staphylococcus aureus infections. Pediatrics 2013;132:e817-24.

22 Matsubara K, Hoshina K, Suzuki Y. Early-Onset and late-onset group B streptococcal disease in Japan: a nationwide surveillance study, 2004-2010. Int J Infect Dis 2013;17:e379-84. 
23 Matsubara K, Hoshina K, Kondo M, et al. Group B streptococcal disease in infants in the first year of life: a nationwide surveillance study in Japan, 2011-2015. Infection 2017;45:449-58.

24 Moore MR, Link-Gelles R, Schaffner W, et al. Effect of use of 13-valent pneumococcal conjugate vaccine in children on invasive pneumococcal disease in children and adults in the USA: analysis of multisite, population-based surveillance. Lancet Infect Dis 2015;15:301-9.

25 Harboe ZB, Dalby T, Weinberger DM, et al. Impact of 13-valent pneumococcal conjugate vaccination in invasive pneumococcal disease incidence and mortality. Clin Infect Dis 2014;59:1066-73.

26 Moore CE, Paul J, Foster D, et al. Reduction of invasive pneumococcal disease 3 years after the introduction of the 13-valent conjugate vaccine in the Oxfordshire region of England. $J$ Infect Dis 2014;210:1001-11.

27 Nakanishi N, Yonezawa T, Tanaka S, et al. Assessment of the local clonal spread of Streptococcus pneumoniae serotype 12F caused invasive pneumococcal diseases among children and adults. $J$ Infect Public Health 2019;12:867-72.

28 Ohkusu M, Takeuchi N, Ishiwada N, et al. Clonal spread of serotype 12F ST4846 Streptococcus pneumoniae. J Med Microbiol 2019;68:1383-90.

29 Ladhani SN, Andrews N, Parikh SR, et al. Vaccination of infants with meningococcal group B vaccine (4CMenB) in England. N Engl J Med 2020;382:309-17.

30 Sard B, Bailey MC, Vinci R. An analysis of pediatric blood cultures in the postpneumococcal conjugate vaccine era in a community hospital emergency department. Pediatr Emerg Care 2006;22:295-300

31 Bressan S, Berlese P, Mion T, et al. Bacteremia in feverish children presenting to the emergency department: a retrospective study and literature review. Acta Paediatr 2012;101:271-7. 